\title{
Constructing a New Nigrostriatal Pathway in the Parkinsonian Model with Bridged Neural Transplantation in Substantia Nigra
}

\author{
Feng C. Zhou, ${ }^{1}$ Yung H. Chiang, ${ }^{1}$ and Yun Wang ${ }^{2}$ \\ ${ }^{1}$ Department of Anatomy and Medical Neurobiology Program, Indiana University School of Medicine, Indianapolis, Indiana \\ 46202, and 2Department of Pharmacology, National Defense Medical Center, Taipei, Taiwan
}

The physical repair and restoration of a completely damaged pathway in the brain has not been achieved previously. In a previous study, using excitatory amino acid bridging and fetal neural transplantation, we demonstrated that a bridged mesencephalic transplant in the substantia nigra generated an artificial nerve pathway that reinnervated the striatum of 6-hydroxydopamine (6-OHDA)-lesioned rats. In the current study, we report that a bridged mesencephalic transplant can anatomically, neurochemically, and functionally reinstate the 6-OHDA-eradicated nigro-striatal pathway. An excitatory amino acid, kainic acid, laid down in a track during the transplant generated a trophic environment that effectively guided the robust growth of transplanted neuronal fibers in a bundle to innervate the distal striatum. Growth occurred at the remarkable speed of $\sim 200 \mu \mathrm{m} / \mathrm{d}$. Two separate and distinct types of dopamine (DA) innervation from the transplant have been achieved for the first time: (1) DA innervation of the striatum, and (2) DA innervation of the pars reticularis of the substantia nigra. In addition, neuronal tracing revealed that reciprocal connections were achieved. The grafted DA neurons in the SNr innervated the host's striatum, whereas the host's striatal neurons, in turn, innervated the graft within 3-8 weeks. Electrochemical volt- ammetry recording revealed the restoration of DA release and clearance in a broad striatal area associated with the DA reinnervation. Furthermore, the amphetamineinduced rotation was attenuated, which indicates that the artificial pathways were motor functional. This study provides additional evidences that our bridged transplantation technique is a potential means for the repair of a completely damaged neuronal pathway.

Key words: excitatory amino acid; neural transplant; dopamine; pathway repair; voltammetry; HRP/immunocytochemistry double staining; rotational behavior
In many neurodegenerative diseases, a breakdown of the neuronal circuity occurs. In such cases, if repair of the disconnected circuit is possible, such repair requires more than intrinsic nerve regeneration and sprouting. More than a decade after neural transplantation was reintroduced, the concept of replenishing degenerated neurons has advanced from basic experimentation to clinical application (Lindvall et al., 1990; Madrazo et al., 1991; Freed et al., 1992; Redmond et al., 1993; Lindvall and Odin, 1994; Kordower et al., 1995). Progress has been made in areas ranging from expanding sources of neuronal tissue to enhancing neuronal survival and nerve fiber outgrowth (Björklund and Stenevi, 1985; Azmitia and Björklund, 1987; Gash and Sladek, 1988; Dunnett and Richards, 1990; Freed, 1993; Rosenstein, 1993; Kordower and Sanberg, 1995). To date, the repair of a completely damaged pathway has not been achieved because of the inability to direct the outgrowth from a transplant. A major obstacle of the homotypic transplant (a graft placed in an ontogenic site) is the lack of outgrowth from the graft reaching to a distal ontogenic target region (Pritzel et al., 1986; Zhou and Buchwald, 1989). Conversely, the ectopic transplant, the most common type of transplant, is placed within its target region and hence suffers by being alienated from its normal afferent connections. In either case, the circuitry is not repaired.

The Parkinsonian model is used for the study of connection

\footnotetext{
Received May 14, 1996; revised Aug. 2, 1996; accepted Aug. 12, 1996.

Correspondence should be addressed to Dr. Feng C. Zhou, Department of Anatomy, Indiana University School of Medicine, 635 Barnhill Drive, Indianapolis, IN 46202.

Copyright (C) 1996 Society for Neuroscience $0270-6474 / 96 / 166965-10 \$ 05.00 / 0$
}

repair, because its symptoms can be induced by the degeneration of a single neuronal type [dopamine (DA)], and its motor pathways have been well studied. In this study, we demonstrated that fetal neuronal transplantation coupled with in situ trophic guiding can physically and chemically repair the damaged circuitry. Recent studies have shown that excitochemicals such as kainic acid (KA) injected into the brain rapidly induce a potent, long-lasting ( $>1$ month) trophic environment that triggers intrinsic sprouting (Takashima et al., 1993; Zhou et al., 1995). We proposed that if the KA-induced trophic effect is strategically confined within a narrow track, it is capable of guiding and facilitating transplanted neurons to send axons through the track to innervate designated distal brain regions (Zhou and Chiang, 1995). The applicability and effectiveness of this bridged transplant in repairing circuitry were evaluated in this study by demonstrating how DA fibers from the graft in response to the excitochemical-induced neurotrophic factors grow in distance, selectively distribute themselves through target and nontarget regions, respond when depolarized, and contribute to the two-way connections.

\section{MATERIALS AND METHODS}

6-OHDA-lesioned and experimental groups. Female Sprague Dawley rats (250-300 gm) were unilaterally injected with 6-OHDA $(5 \mu \mathrm{g} / \mu \mathrm{l})$ by two injections (6 $\mu \mathrm{l}$ each) into the ascending mesostriatal pathway $(4.2 \mathrm{~mm}$ posterior to bregma, $1.1 \mathrm{~mm}$ lateral to the midline, $7.8 \mathrm{~mm}$ below the dura and $4.4 \mathrm{~mm}$ posterior to bregma, $0.9 \mathrm{~mm}$ lateral to the midline, $7.8 \mathrm{~mm}$ below the dura) near the medial forebrain bundle to remove DA innervation to the striatum. These routine lesions removed $\sim 95 \%$ of the DA innervation. As examined by immunocytochemistry for tyrosine hydroxylase, no reinnervation occurred after lesioning in five rats in $>3$ months. Routine rotational behavior tests were preformed to screen for rats with 
$>300$ turns/hr after 6-OHDA lesioning (animals were tested $14 \mathrm{~d}$ after lesioning using a rotometer as described in Hudson et al., 1993); at this stage, primarily ipsilateral turning was observed after amphetamine challenge ( $5 \mathrm{mg} / \mathrm{kg}$, i.p.) for selection as candidates for bridged transplantation. Fourteen to $21 \mathrm{~d}$ after the 6-OHDA-lesioning, animals were bridgetransplanted (see below) from the substantia nigra (SN) to the striatum with fetal mesencephalic tissue.

Six animals were killed for immunocytochemistry at 3 weeks after transplantation to evaluate early fiber growth in the track and in the striatum, and the remaining rats were used for various assays between 4 and 8 weeks after transplantation. Twelve rats were used for $\mathrm{TH}$ immunocytochemical staining of the striatum and transplants in the SN, 7 for HRP-track tracing, and 12 for in vivo electrochemical analysis (6 for DA release and 6 for DA uptake). Two groups of animals were specifically designed to test rotational behavior before and after the bridged transplant: (1) in the experimental group, the bridge was laid down between the SN and the striatum $(n=8)$; (2) in the control group, the bridge was laid down from the SN through the lateral ventricle to the striatum $(n=6)$. (We incidentally found that the lateral ventricle breaks the continuum of the bridged pathway and serves as a good control.)

Transplant and excitochemical injection. Sprague Dawley rats were used as both hosts and donors. The hosts were ipsilaterally transplanted with fetal mesencephalic tissue into the SN 14 to $21 \mathrm{~d}$ after 6-OHDA lesioning. Donor fetal mesencephalic tissue was freshly obtained from embryonic day 14 (E14) fetal brains. A one-step injection method was used in this study to introduce fetal tissue and lay down the KA track (for details, see Zhou and Chiang, 1995). Briefly, both tissue slurry and KA were injected through a glass micropipette according to the following sequence: $4 \mu \mathrm{l}$ of mesencephalic slurry $\left(\sim 0.5 \times 10^{5}\right.$ cells $), 1 \mu \mathrm{l}$ air, and $4 \mu \mathrm{l} \mathrm{KA}(0.25 \mu \mathrm{g} / \mu \mathrm{l})$ laying down a track either from the SN to the striatum $(9.8 \mathrm{~mm}$ anterior to bregma, $2.5 \mathrm{~mm}$ lateral to the midline, $15.8 \mathrm{~mm}$ below the dura, and $60^{\circ}$ from vertical) or via the lateral ventricle to the striatum as a control $(8.2$ $\mathrm{mm}$ anterior to bregma, $2.5 \mathrm{~mm}$ lateral to the midline, $15.8 \mathrm{~mm}$ below the dura, and $60^{\circ}$ from vertical). We found that all control transplants with this track fell into the lateral ventricle and did not have satisfactory innervation to the $\mathrm{SNr}$ and striatum. The fetal mesencephalic tissue was delivered at the $\mathrm{SN}$ over a period of $5 \mathrm{~min}$; the needle was slowly retracted while the air was released. This was followed by the injection of KA over a 5 min period, evenly distributing the KA through the track between the SN and the striatum. The animals were killed 3 weeks after transplantation for DA fiber staining in the track or 6-8 weeks after transplantation for DA fiber staining in the striatum, HRP track tracing, or voltammetry.

Immunocytochemistry. All animals selected for immunocytochemistry were perfused intracardially with formaldehyde (reagent grade) freshly made from $4 \%$ paraformaldehyde and $0.1 \mathrm{M}$ phosphate-buffer (PB) under deep ketamine cocktail anesthesia (1000 mg of ketamine, $22 \mathrm{mg}$ of ace promazine, and $4.8 \mathrm{mg}$ of atropine in $10 \mathrm{ml}$ solution; $120 \mathrm{mg} / \mathrm{kg}$, i.p.). A $20 \mathrm{~min}$ perfusion of $10 \%$ sucrose in the same fixative followed. Their brains were removed, left in the same fixative plus $30 \%$ sucrose overnight, and sagittally sectioned at 40 $\mu \mathrm{m}$ with a cryostat for immunocytochemical staining (Zhou et al., 1991, 1994). The Sternberger peroxidase-antiperoxidase (PAP) indirect-enzyme method was used for staining. The PAP reaction was done with $0.003 \% \mathrm{H}_{2} \mathrm{O}_{2}$ and $0.05 \%$ 3'3-diaminobenzidine (DAB). The primary, secondary, and marker antibodies were diluted with phosphate-buffered saline (PBS) containing $1 \%$ normal sheep serum. The primary antibody anti-tyrosine hydroxylase (TH, Pel Freez and Eugene Tech) was incubated overnight, the corresponding second and third antibodies for $1 \mathrm{hr}$ each. Three washes with PBS, each for $5 \mathrm{~min}$, were used between each antibody incubation.

HRP tracing doubled with immunocytochemical staining. A 30-100 nl microinjection of $5 \%$ wheat germ agglutinin-HRP (in saline) was gaspressure- or gauge-delivered into the dorsal striatum $(0.5 \mathrm{~mm}$ anterior to bregma, $2.5 \mathrm{~mm}$ lateral to the midline, $5.5 \mathrm{~mm}$ below the dura) of rats that received the grafts. Twenty-four hours later, all animals were perfused with fixative freshly made from $4 \%$ paraformaldehyde in $\mathrm{PB}$, followed by $10 \%$ sucrose in PBS. The brains were blocked around the injection site and stored in $30 \%$ sucrose until they sank. The blocks were then sectioned of $40 \mu \mathrm{m}$ with a cryostat microtome. The HRP color reaction (according to Mesulam, 1978) was carried out by incubation of the tissue in $0.05 \%$ tetramethyl benzidine (TMB, Chromogen) for $20 \mathrm{~min}$, transferred through three distilled water washes of $5 \mathrm{sec}$ each, followed by 10 $\min 0.01 \% \mathrm{H}_{2} \mathrm{O}_{2}$ reaction, and then washed three times in distilled water. At the end of the reaction, the TMB reaction products were stabilized by incubating the tissue for $15 \mathrm{~min}$ in stabilizing solution containing $0.05 \%$ DAB, $1 \%$ cobalt chloride, $1 \%$ nickel chloride, and $0.01 \% \mathrm{H}_{2} \mathrm{O}_{2}$, followed by three transfers in PBS for 5 min each. The HRP reaction products were black and granular in form.

The stained tissue was then transferred into anti-TH sera for immunocytochemical staining. The PAP indirect-enzyme method was used, as described as above. The immunostained products were brown in color and homogeneous in form. The HRP-containing neurons were considered positive when the black HRP granules exceeded 20 (signal-to-noise ratio, 20:1). Most of the HRP retrogradely labeled neurons in the transplant contained many HRP granules packed in the cytoplasm.

Electrochemical methods. Rats were anesthetized with urethane $(1.25$ $\mathrm{gm} / \mathrm{kg}$, i.p.), intubated, and placed in a Kopf stereotaxic apparatus. A portion of the skull extending from $1 \mathrm{~mm}$ anterior to $4 \mathrm{~mm}$ posterior to bregma and 1-4 $\mathrm{mm}$ lateral to the midline was removed for electrode recording. Remote from this site, a miniature $\mathrm{Ag} / \mathrm{AgCl}$ reference electrode was inserted into the brain and cemented in place with dental acrylic. In vivo chronoamperometric measurements of extracellular DA concentrations were performed with a microcomputer-controlled apparatus (IVEC-10, Medical Systems, Greenvale, NY). The recordings were taken at a continuous rate of $10 \mathrm{~Hz}$ throughout the experiment, using Nafion-coated (5\% solution, Aldrich Chemical, Milwaukee, WI) carbon fiber working electrodes. These electrodes have been shown to be highly sensitive for monoamine neurotransmitters (Gerhardt et al., 1984). An oxidation potential of $+0.55 \mathrm{~V}$ for $50 \mathrm{msec}$ (square-wave pulses), relative to an $\mathrm{Ag} / \mathrm{AgCl}$ reference electrode, was applied at a rate of $10 \mathrm{~Hz}$. The resulting oxidation current was integrated during the last $80 \%$ of the pulse. The current generated during the reduction of the oxidized electroactive species was digitized in the same manner when the potential dropped back to its resting level $(0 \mathrm{~V})$. The linearity and sensitivity of all electrodes used in the in vivo experiments were determined using DA standard solutions in vitro ranging from 2.0 to $10.0 \mu \mathrm{M}$. For calibration curves for DA sensitivity and DA, ascorbic acid selectivity ratios were determined for all electrodes before their use. Only electrodes exhibiting highly linear response $(r>0.997)$ and selectivity $(>500: 1$, compared with ascorbic acid) to DA were used for the in vivo experiments (Gerhardt and Palmer, 1987; Wang et al., 1994). The ratios of reduction-to-oxidation currents, at the peak of the oxidation signal, were used as an index to qualitatively identify whether the compound measured was DA. (All in vivo signals were expressed as micromolar changes in $\mathrm{DA}$ using in vitro calibration curves.)

The release and clearance of DA was measured by the changes in extracellular DA concentration after microinjection of $\mathrm{KCl}$ (six animals) or DA (six animals), respectively, in different animals, into the striatum. $\mathrm{KCl}(70 \mathrm{~mm}, 100-200 \mathrm{nl})$ or DA $(200 \mu \mathrm{M}, 150-200 \mathrm{nl})$ was applied locally through a multibarrel pipette. The working electrode and the multibarrel micropipette were mounted together with sticky wax (Kerr, Sybron, CA); the tips were separated by $200 \mu \mathrm{m}$. The electrode/pipette assembly was lowered into the striatum $(1.5 \mathrm{~mm}$ anterior to $0.5 \mathrm{~mm}$ posterior to bregma, $1.8-4 \mathrm{~mm}$ lateral to the midline, and $4.0-7.0 \mathrm{~mm}$ below the dura). Local application of drugs from the multibarrel micropipette was performed by pressure ejection using a pneumatic pump (PPM-2, Medical Systems, Great Neck, NY). The ejected volume was monitored by recording the change in the fluid meniscus in the pipette before and after ejection by using a dissection microscope. Three to four tracks were used, and 10 to 12 recordings were made in each animal.

Amphetamine-induced rotation. The animals were tested for rotational behavior $14 \mathrm{~d}$ after lesioning and 6-8 weeks after transplantation in a multichannel rotometer (Hudson et al., 1993) in a quiet, dimly lit room. The rotometer consists of several $50 \mathrm{~cm}$ diameter plastic bowls, each with a harness attachment connected to the computer to record clockwise or counterclockwise turns. A full $360^{\circ}$ turn in one direction was considered a turn. Animals with unilateral 6-OHDA lesioning with our lesioning paradigm ( $>95 \%$ denervation as detected by anti-TH immunostaining) have shown an ipsilateral rotation with $5 \mathrm{mg} / \mathrm{kg}$, i.p., amphetamine challenge. A 90 min rotational measurement after each amphetamine injection was performed. The highest block of turns in $60 \mathrm{~min}$ was chosen by the computer program for comparison and expressed in a unit of turn/min or turn/hr. Animals were tested twice after lesioning and twice after transplantation, and an average was taken for each time point.

\section{RESULTS}

As described previously, the excitatory amino acid KA, known to kill adult neurons at high dosage, did not damage the fetal neurons in a morphologically observable manner. Also, at the dose used, little damage to the host neurons along the track was 
observed. All transplanted animals had a sizable graft $(>2 \mathrm{~mm}$ in diameter) in which many TH-positive and methyl greencounterstained neurons (Nissl positive, $>13 \mu \mathrm{m}$ ) were found. We noticed two morphologically different populations of TH-positive cells clustered in the transplant. Here, we report that large cell bodies are associated with many thick dendrites and small cell bodies with sparse, thin processes. By examining serial sections and measuring the size of $120 \mathrm{TH}$-positive neurons with their nuclei shown on the sections from six animals (with computeraided image analysis), we found that the group of small neurons were $16 \times 10 \mu \mathrm{m}$ in diameter and $134 \pm 3 \mu \mathrm{m}^{2}$ in area and the large neurons $31 \times 15 \mu \mathrm{m}$ in diameter and $371 \pm 17 \mu \mathrm{m}^{2}$ in area. The large DA neurons could be nigral DA neurons, whereas the small neurons could be of ventral tegmental origin. The two DA populations were also observed in monkey mesencephalic transplants (J. Sladek, personal communication). Retrograde tracing indicated that both types of neurons projected to the striatum through the track, as described below.

\section{Reciprocal connections}

The HRP injection site was small and primarily covered the rostral third of the dorsal striatum. It diffused along the needle track and was confined to the cortex immediately above the injection site after $24 \mathrm{hr}$. The actual size of the HRP absorption site is smaller than that at $24 \mathrm{hr}$ of diffusion and is closer to that of the first $2 \mathrm{hr}$ of diffusion (Mesulam, 1978; Zhou et al., 1994). In conjunction with TH immunocytochemistry, four types of neurons were observed: TH-positive (brown homogeneous DAB staining), HRP-positive (with $>20$ black granules in the cell bodies), THand HRP-positive, and nonlabeled neurons.

Two types of reciprocal innervations were identified (Fig. 1d,e). First, the retrogradely transported HRP was detected in THpositive neurons (black granules in brown DAB-colored cells) as well as in non-TH-positive neurons in the grafts, which indicates that fetal DA and non-DA neurons in the grafts projected to the striatum. There were no HRP-/TH-double-positive nor THpositive cells in the degenerated $\mathrm{SN}$, which indicates that DA innervation in the striatum derived from DA neurons in the graft not the degenerated SN. Many neurons in the grafts were HRPpositive and TH-positive (in two small HRP injection sites confined in the dorsal striatum and in tract at adjacent rostral cortex, $\sim 196$ of 1432 TH-positive neurons are also HRP-positive in one transplant and 192 of 1893 in another transplant), whereas single HRP- and TH-positive neurons were also found. The HRP injection site was small and did not cover the entire dorsal striatum, thus the HRP-labeled neurons in the graft represent only part of the population that projected to the striatum. This indicates that there are more DA as well as non-DA graft-striatal projections through the bridged track to the striatum than we observed with the current detection methods.

Second, many antegrade-transported HRP striatal cell bodies were identified, and their projections were traceable along the internal capsule to the medial forebrain bundle and their terminals were found in the graft. This indicates that the striatonigral fibers grew into the graft to make contacts. The immunocytochemical staining of DA fibers plus the retrogradely and antegradely transported HRP staining together indicates that reciprocal projections were achieved. The transplanted DA neurons in the SN sent out major DA fibers to the striatum, and the striatal neurons also projected into the graft (Fig. 3).

\section{Dual innervation and recapitulated fetal DA innervation in the adult striatum}

Two separate and distinct types of DA innervation (detected by TH immunocytochemistry) were evident: (1) long, far-reaching DA fibers extended along the KA track to the striatum (Fig. 1; $>80 \%$ of the immunostained bridged transplants have $\mathrm{TH}-$ positive fibers extending from transplants into the bridges), and (2) short DA fibers surrounded the graft in the $\mathrm{SN}$ and projected a short distance into the pars reticularis $\mathrm{SNr}$ (Fig. 2; of 18 transplants, 14 had TH-positive axonal fibers extended into the pars reticulata). The contrast between the two types of fiber outgrowth reveals the remarkable effects of KA on fiber extension. At the KA injection site, dense TH-positive fibers extended characteristically straight and unbranching from the fetal DA neurons and grew straight along the KA track, as reported previously (Zhou and Chiang, 1995). They formed a dense bundle of $\sim 150 \mu \mathrm{m}$ in diameter and extended several millimeters into the striatum (Fig. 1). In contrast, on the caudal side of the graft, without a KA tract, the TH-positive fetal DA fibers grew into the surrounding nigra; they were short and smaller in scale. DA innervation from the transplant to the $\mathrm{SNr}$ was observed (Fig. 2).

The growth rate of the fetal DA fibers in the KA track was remarkable. Of six animals sacrificed at 3 weeks, TH-positive fibers had already reached the striatum through the KA track. The average growth rate was $\sim 200 \mu \mathrm{m} / \mathrm{d}$ along the KA track. Our unpublished observations indicate that fetal neurons do not send out fibers within the first $5 \mathrm{~d}$ after transplantation. With this consideration in mind, the estimated growth rate could increase to $300-400 \mu \mathrm{m} / \mathrm{d}$.

The straight, unbranching, and tightly bundled DA fibers remained in the track through the thalamus and globus pallidus but spread out of the track as soon as they reached the striatum (Zhou and Chiang, 1995) (Fig. 1). In this study, we found that the pattern of DA innervation in the striatum was unique. As early as 3 weeks after transplantation, the striatum exhibited a patchy TH-positive fiber distribution around the KA track (Fig. 1). This patchy distribution was transient and became indistinct later as $\mathrm{TH}$ positive fibers filled in the gaps at $6-8$ weeks after transplantation. TH-positive fibers could be traced leaving the track and innervating a large area of the striatum. The distribution of TH-positive fibers was characteristically dense proximal to and sparse distal to the track, indicating a growth gradient away from the main fiber bundles in the track.

In contrast, the grafts of control rats with a track from the $\mathrm{SN}$ through the lateral ventricle to the striatum were found to have few TH-positive fibers innervating either the striatum or the SNr. However, because the KA-bridged track passed through the ventricle, there were many $\mathrm{TH}$-positive neurons in the lateral ventricle and a great number of TH-positive fibers innervating the ependymal layer (data not shown).

The establishment of new connections led to the question of whether ingrowing fibers release DA in the striatum. DA release was induced by the local application of $\mathrm{KCl}(70 \mathrm{~mm}, 125 \mathrm{nl})$ (Fig. 4, arrows) into the striatum. Voltammetric analysis revealed that $\mathrm{K}^{+}$induced DA overflow, abolished in the 6-OHDA-lesioned striatum, was detected in a millimeter-wide area of the striatum of the transplanted animals (Fig. $4 A$ ). The peak of DA overflow induced by $\mathrm{K}^{+}$ stimulation was restored in all of the transplanted animals tested. An average $3 \times 3 \times 2 \mathrm{~mm}$ area of the striatal field responded to $\mathrm{K}^{+}$and released DA. Higher DA release was found in an area near the KA track and lower DA release distal to the track (Fig. $4 C$ ). Longer time 

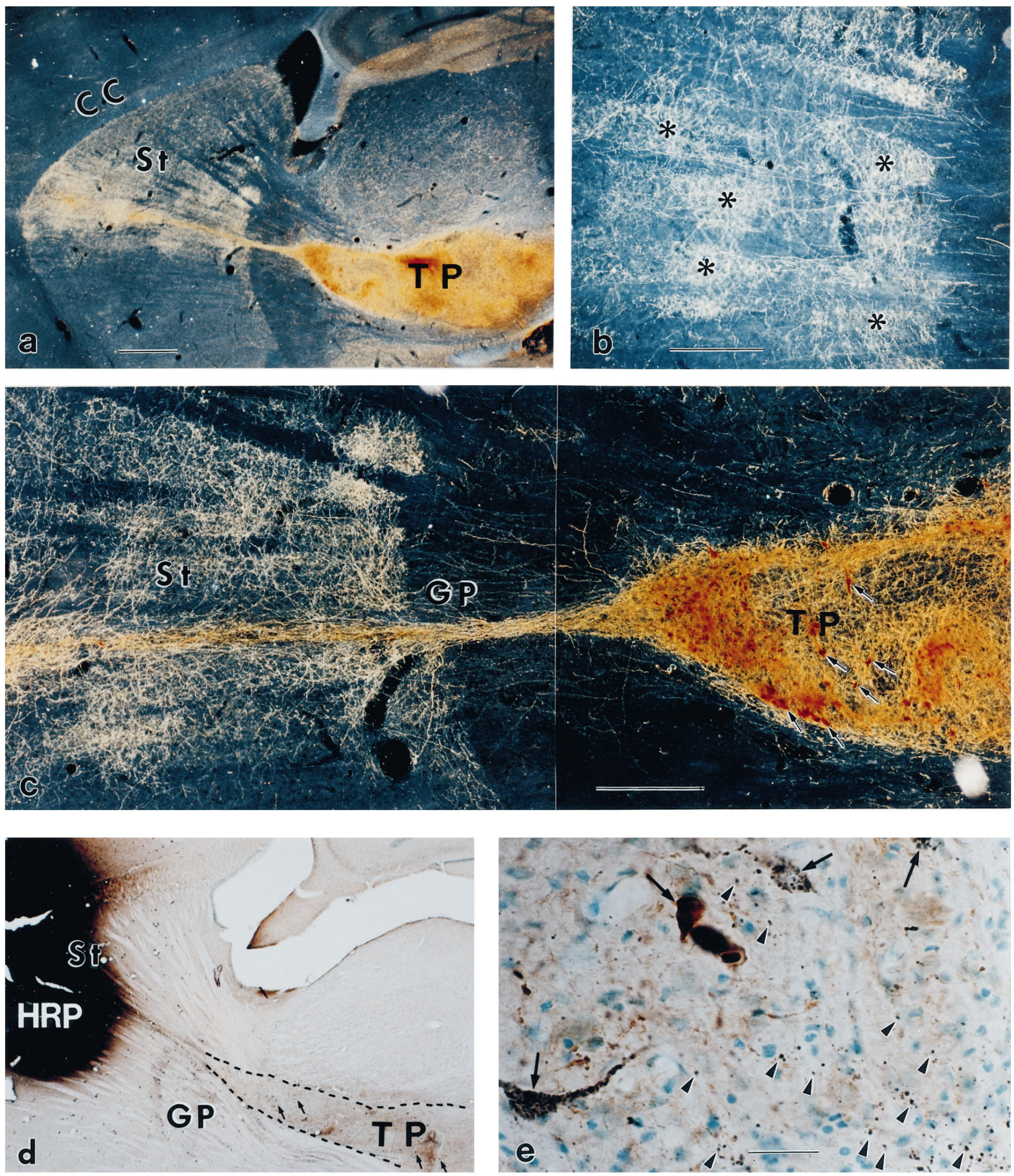

Figure 1. Striatonigral and nigrostriatal reconnections by the KA-bridged mesencephalic transplant. $a$, The TH-positive neurons in the bridged mesencephalic transplant $(T P)$ sent bundled fibers along the KA track through the globus pallidus $(G P)$. These fibers from the bundle expand immediately on arrival in the striatum (St). b, On arrival, the TH-positive fibers form patches (asterisks) in the striatum, which is similar to the developmental pattern of DA fibers in the immature St at midgestation. The patchy innervation $(b)$ is transient and spreads into homogeneous innervation $(c)$ (Zhou and Chiang, 1995) over a 3-6 week period after transplantation. The TH immunocytochemistry (brown) and HRP (black) double staining indicate that HRP injection in the St and the adjacent cortex $(d)$ results in retrograde transport labeling of the neuronal cell bodies in the TP; many of them are HRP- and TH-double-labeled (e, arrows) (in this small injection site, $1432 \mathrm{TH}$-positive neurons were observed in transplant; among them $\sim 13.7 \%$ were 

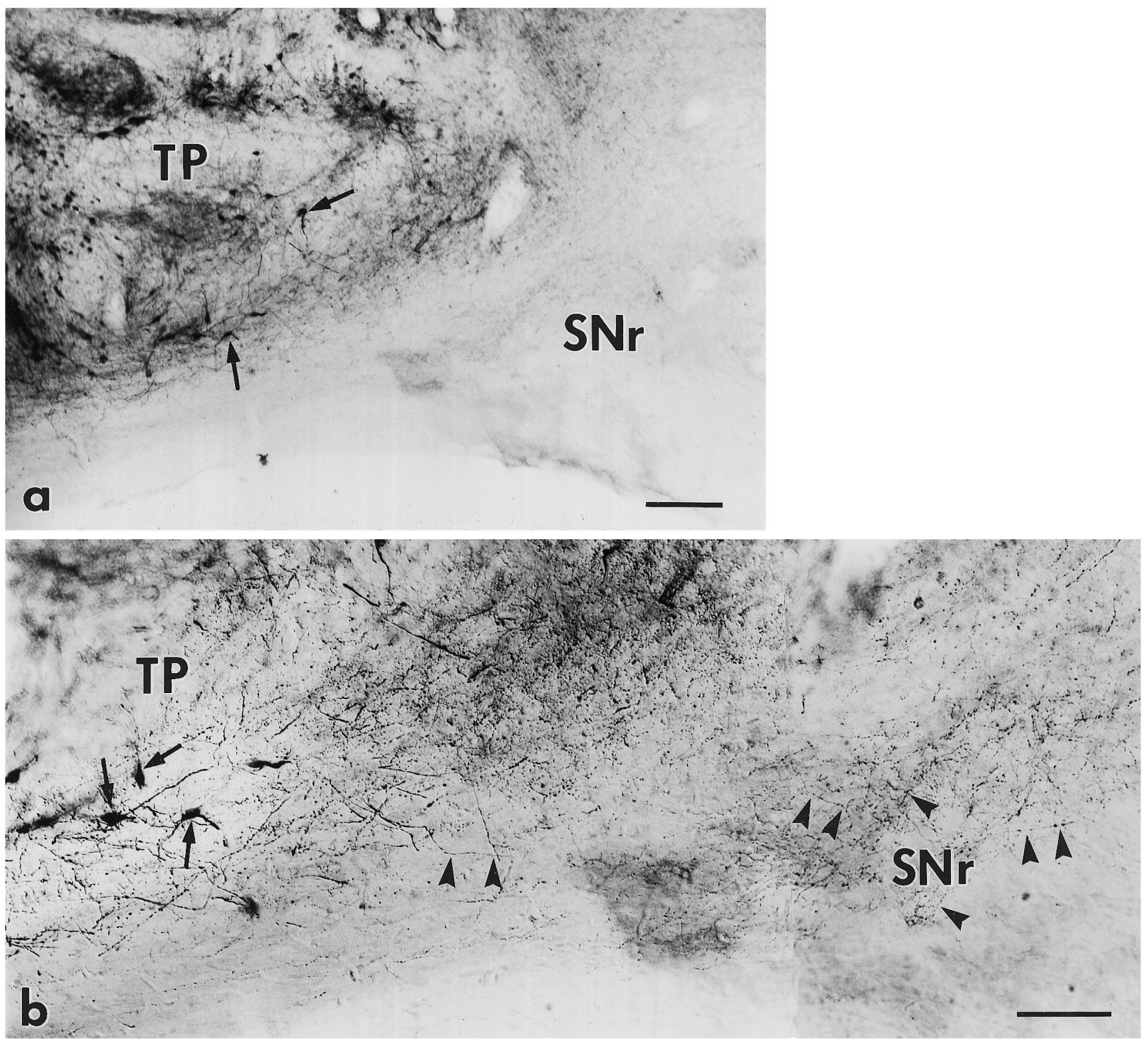

Figure 2. The TP placed in the SN not only sent long DA fibers innervating the striatum but also sent short DA innervations to the functionally important SNr. A mesencephalic transplant near the nigra is shown in $a$ containing many TH-positive neurons (arrows). The fibers of DA neurons in the transplant grew a short distance into the $\mathrm{SNr}$, where major striatonigral terminals and GABAergic output neurons reside. Characteristic varicosities decorating thin TH-positive fibers (arrowheads; $b$, enlargement of bottom third of $a$ ) are seen in the host SNr. Bright field; the background is Nissl-counterstained with methyl green. Scale bars: $a, 200 \mu \mathrm{m} ; b, 100 \mu \mathrm{m}$.

course studies are required to address whether functional DA release persists over longer survival period.

DA clearance that was abolished in the 6-OHDA-lesioned striatum was restored after the KA-bridged transplant. Local application of $200 \mathrm{nl}$ of $200 \mu \mathrm{M} \mathrm{DA} \mathrm{(Fig.} \mathrm{4B,} \mathrm{arrows)} \mathrm{to} \mathrm{the}$ control nonlesioned striatum resulted in a short DA retention $\sim 6$ $\mu \mathrm{M}$ (Fig. 4B2). Similar to previous findings, the retention of an extracellular DA concentration in the striatum was greater and prolonged after 6-OHDA lesioning (Wang et al., 1994) (Fig. 3B2 vs $B 1)$. Bridged grafts significantly reduced DA retention after exogenous application of DA in the reinnervated striatum (Figs. $3 B 3,4 D)$.

\section{Functional correlation}

Rotational behavioral data show that amphetamine challenge induces a body distortion toward the lesion side and induces

HRP-positive). The TH and HRP double labeling of the cell bodies in the TP indicate that DA neurons in the bridged TP sent fibers to the St and/or cortex. The anterograde transport labeling also results in punctate terminal staining in the TP (e, arrowheads). These punctate HRP granules are located outside the TH-positive fibers, indicating that they are not retrogradely transported labeling through DA fibers but rather antegradely transported through fibers of striatal neurons into their terminals within the TP. This suggests that the striatal neurons also sent fibers that terminate in the TP. $a-c$, Dark-field photographs; $d, e$, bright-field photographs. $C C$, Corpus callosum. Scale bars: $a, d, 1 \mathrm{~mm} ; b, c, 0.5 \mathrm{~mm} ; e, 30 \mu \mathrm{m}$. 


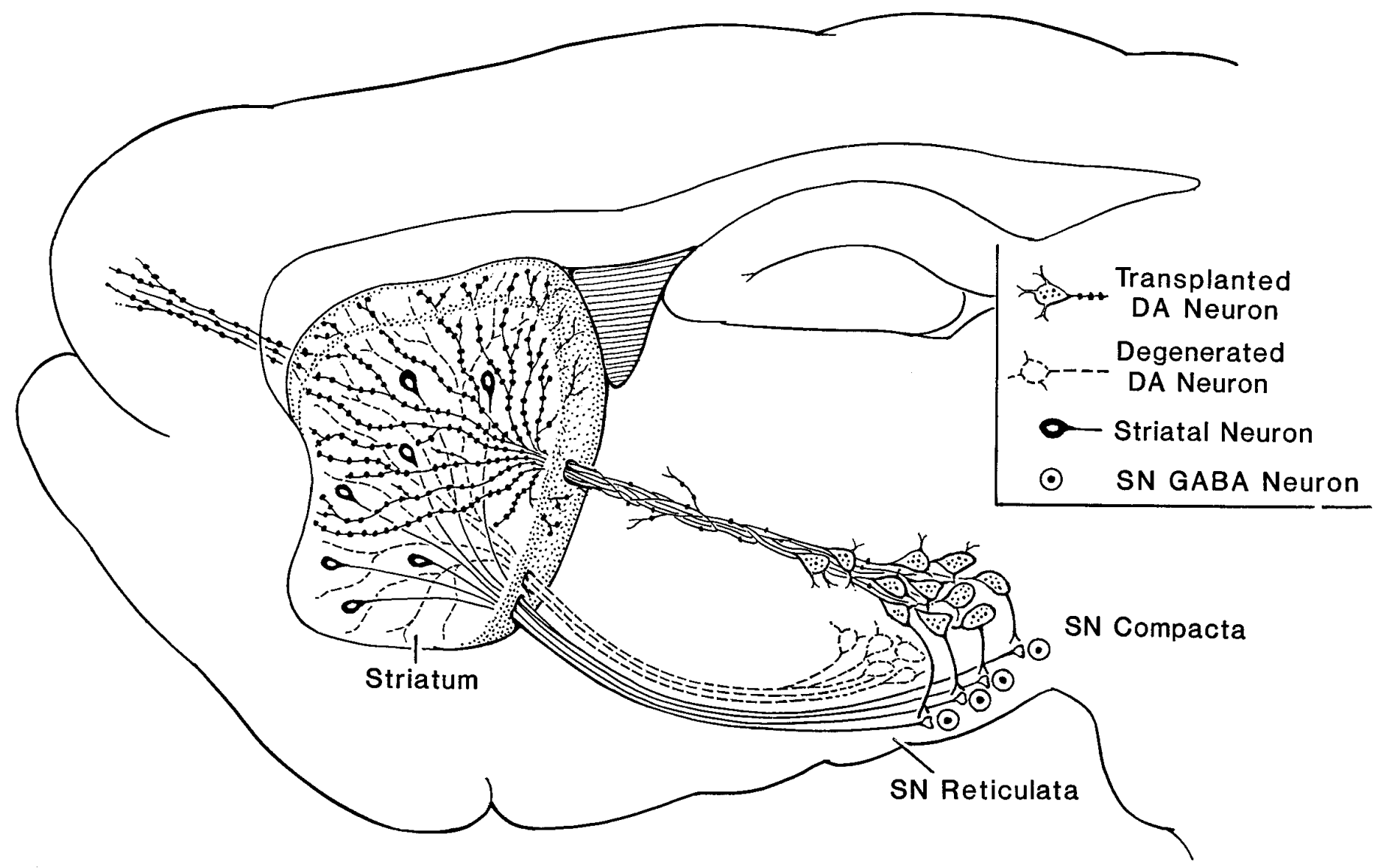

Figure 3. The drawing shows the artificial bridge reconnecting three disrupted sites in the major loop of the basal ganglial motor circuitry. 6-OHDA removes the DA neurons in the SN pars compacta (dotted lines) as in Parkinsonism, which removes the DA regulation of distal striatal neuronal cell bodies, and local striatonigral terminals (which regulate the GABA output neurons in the SNr). The bridged transplant through distal and local reinnervations restores the DA connections to influence the above two sites and may also receive signals from the striatonigral neurons.

ipsilateral rotations in 6-OHDA-lesioned animals (Hudson et al., 1993). In the KA-bridged transplant (nigra to striatum) group, body distortions of the animals were mild or disappeared, and the number of rotations were significantly reduced, but the rotational patterns were complex (Fig. 5). Three types of turning patterns were observed: (1) a significant reduction in ipsilateral turning, evident in animals with bridge transplantation, (2) a mixture of low ipsi- and contralateral rotation, (3) rotations toward the contralateral side, shown by some animals (Fig. 5). The contralateral rotations seem to be a transient phenomenon, because they were reduced along the time course of the $90 \mathrm{~min}$ recording. The contralateral turnings were most significant within the first $30 \mathrm{~min}$ of the test. However, in the control (nigra to lateral ventricle) group, there were no significant differences in amphetamineinduced rotation before or after transplantation (Fig. 6).

\section{DISCUSSION}

The survival of our robustly growing transplanted fetal neurons may be attributable to (1) a lack of NMDA or AMPA receptors, which spares the neurons from a direct $\mathrm{Ca}^{2+}$ influx and/or (2) a lack of glutaminergic innervation to the fetal neurons, which prevents a large secondary $\mathrm{Ca}^{2+}$ influx (Choi, 1994). The noticeably minor damage to the host tissue, confined within the thin track, could be the result of a light KA dosage and/or humoral trophic protection derived from the fetal tissue. KA injected into the brain is known to increase nerve growth factor, glial cell-derived neurotrophic factor, and neurotrophin mRNA transcription and protein expression (Zafra et al., 1990; Ballarin et al., 1991; Humpel et al., 1995). Also, we have found that KA induces a trophic environment in $<1$ week (Zhou et al., 1995).

Figure 4. Transplantation of fetal mesencephalic tissue into the SN followed by KA bridging from the SN to the striatum restores the release and clearance of DA in the striatum 6-8 weeks after transplantation. A1, Local application of $\mathrm{KCl}(70 \mathrm{mM}, 125 \mathrm{nl})$ (arrows) to the control nonlesioned striatum induces DA release. $\mathrm{KCl}$ is applied locally through a multibarrel pipette placed in the striatum of the rat. Extracellular DA concentration is recorded using Nafion-coated carbon fiber electrode. Upper trace, $O X$ represents the extracellular signal from DA oxidation. The ratios of reduction (lower trace, $R E D)$ to oxidation currents are used to qualitatively identify the electroactive species as DA. $A 2, \mathrm{~K}^{+}$-induced DA overflow is abolished in the lesioned striatum and is restored in the lesioned striatum with a KA-bridged transplant (nigra to striatum) $(A 3)$. The peak of DA overflow (mean \pm SEM) induced by $\mathrm{K}^{+}$ stimulation is restored in the 6-OHDA-lesioned striatum from six transplanted animals. B1, Local application of DA (200 $\mu \mathrm{M}, 200 \mathrm{nl})$ (arrows) to the control nonlesioned striatum resulted in a retention of $\sim 6 \mu \mathrm{M}$ DA overflow. B2, The extracellular DA concentration is increased after a 6-OHDA lesion 
A DA Release in the Striatum
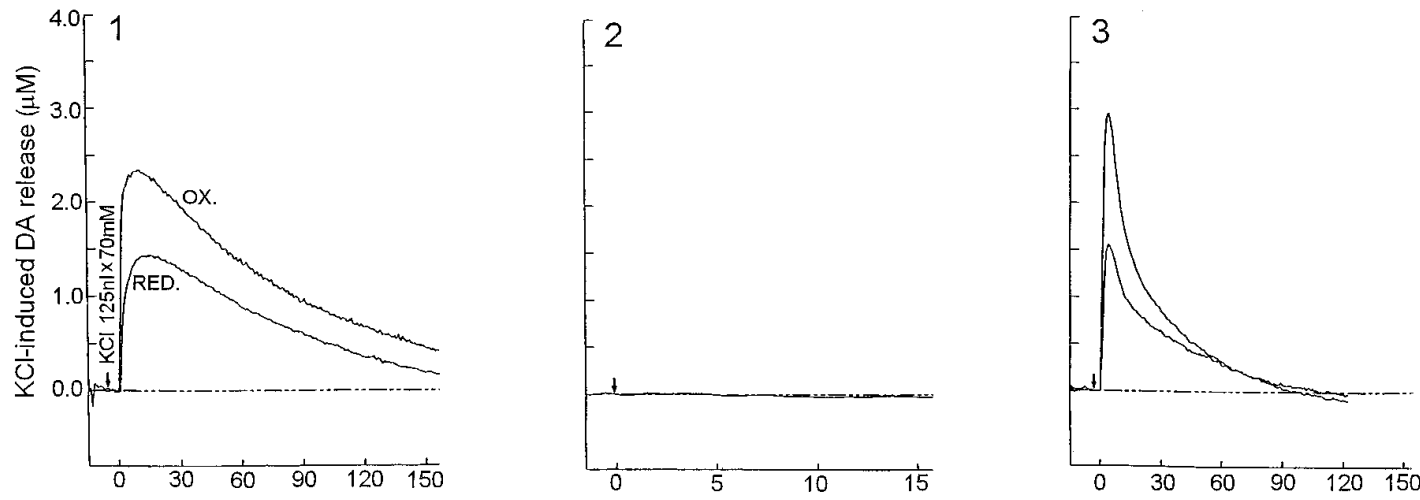

B DA Clearance in the Striatum
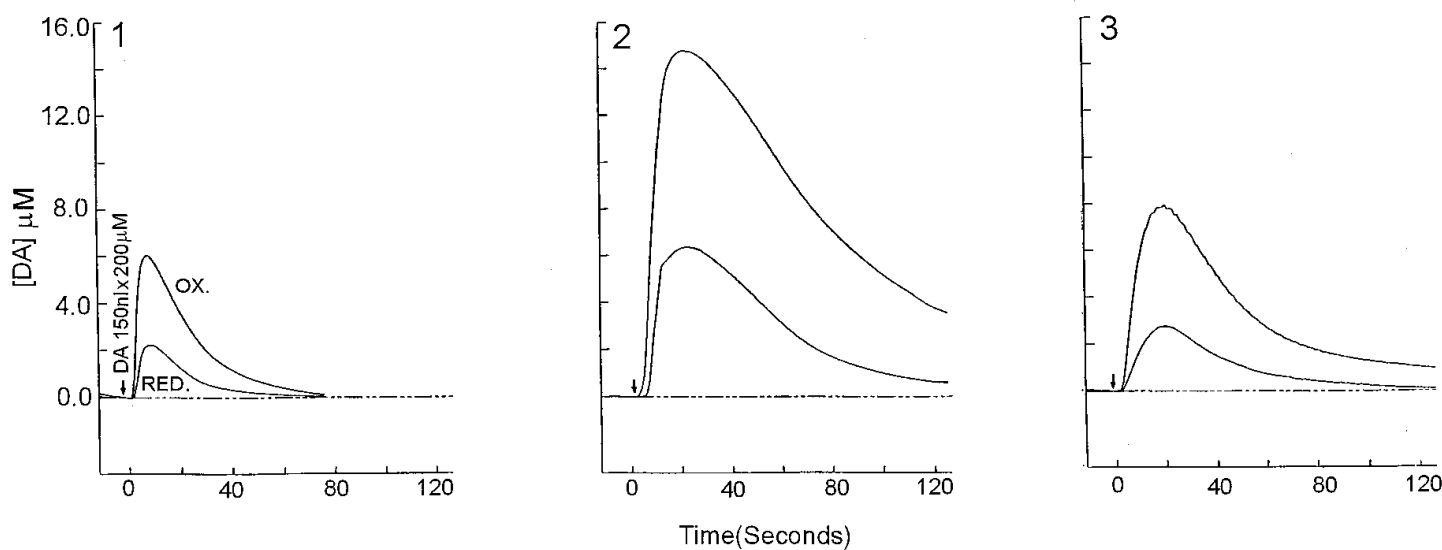

C

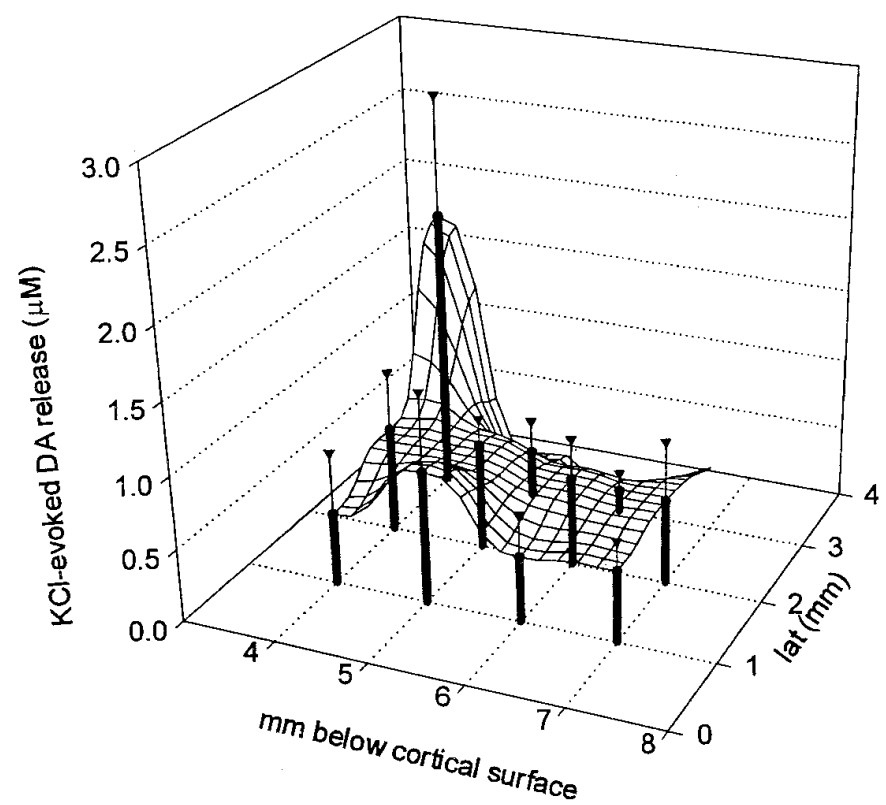

D

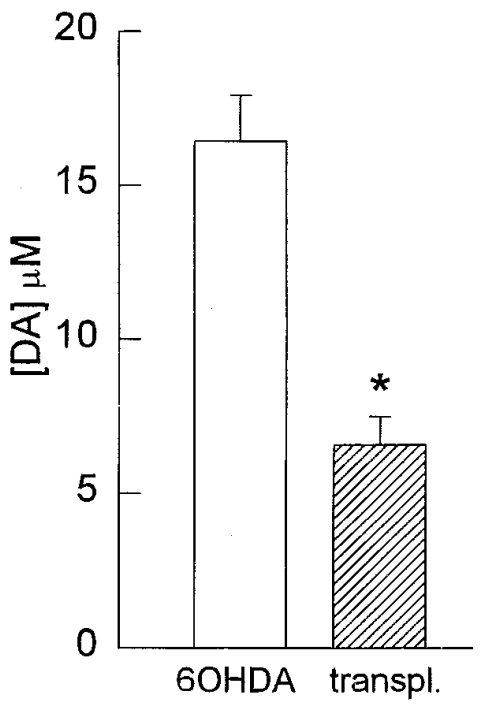

( $B 2$ vs $B 1)$ and is reduced in the lesioned striatum with a KA-bridged transplant (B3). $C$, A much broader area of DA release in the lesioned striatum is achieved by the KA-bridged transplant. A $2 \times 3 \times 3 \mathrm{~mm}$ striatal field is found responsible to $\mathrm{K}^{+}$to release DA, with a higher release near the needle track. $D$, Restoration of DA clearance in transplanted animals. Open and filled bars represent peak extracellular DA concentrations in the lesioned and bridged striatum from six animals, respectively. The KA-bridged transplant diminishes the increase in DA overflow induced by local application of DA into the lesioned striatum $(* p<0.01, t$ test $)$. 


\section{Bridged Transplantation on Rotation Behavior of Semiparkinsonian Rats Before Transplant}

a.

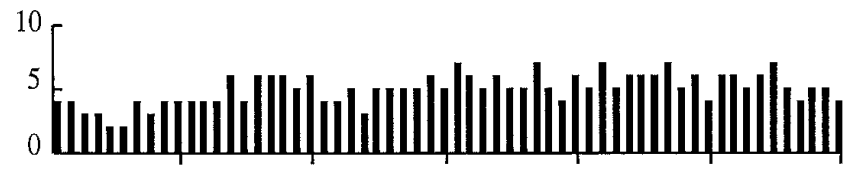

e.

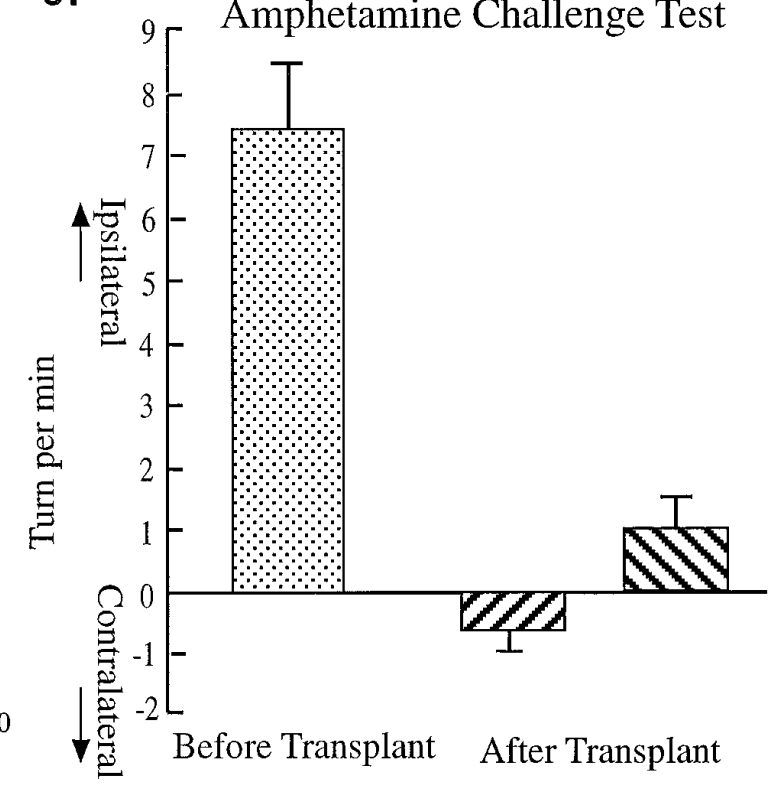

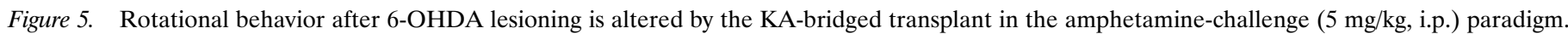

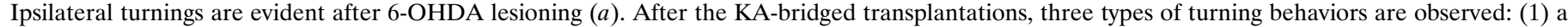

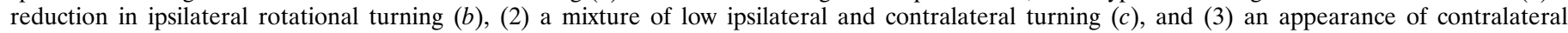

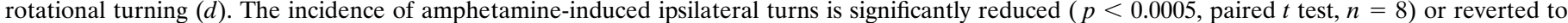
contralateral turning (4 of 8 ) after bridged transplant $(e)$.

In this study, robustly growing transplanted fetal neuronal fibers were effectively guided by the KA bridge to reinnervate the 6-OHDA-lesioned striatum over the course of 3-8 weeks. A unique feature of the bridged fibers is that they remained straight,

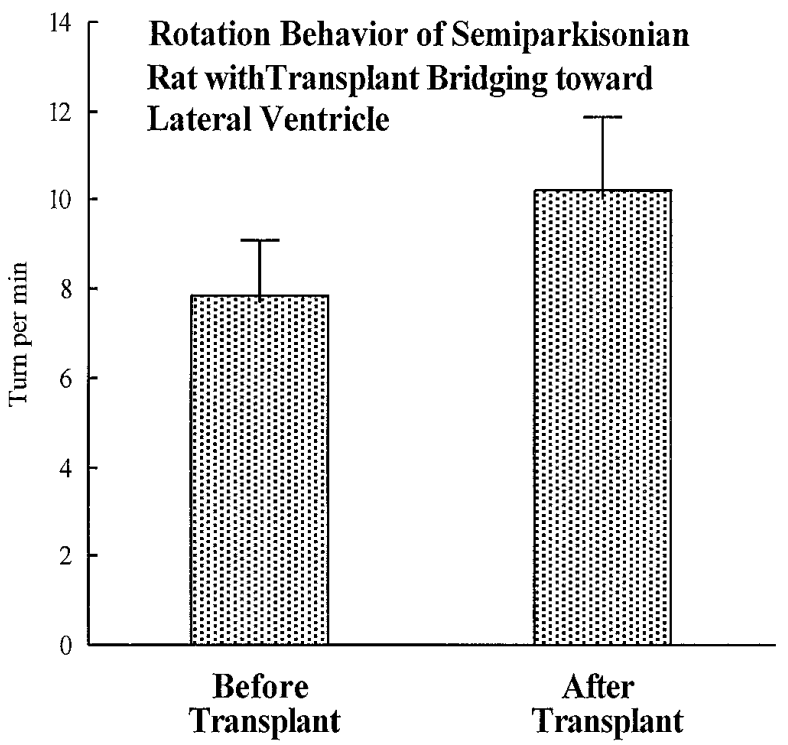

Figure 6. Control animals receiving the KA-bridged transplant from the SN through the lateral ventricle to the striatum show no changes in rotational behavior. The amphetamine-induced rotational behavior of these rats shows no improvement after transplantation $(p>0.05$, paired $t$ test, $n=6$ ). Immunocytochemistry also indicates that little innervation to either the striatum or the SNr has been achieved. unbranching, and tightly bundled within the track throughout the thalamus and globus pallidus but spread out of the track as soon as they reached the striatum (Fig. 1). Additionally, when the long, extending KA injection-guided DA fibers reached the striatum, a selective pattern of innervation was observed. This presents us with a unique opportunity to address an unanswered question: How will the transplanted fetal DA fibers be distributed in the cytoarchitecturally mature striatal mosaic? (A testing paradigm is not available through local striatal transplantation that is confounded by adjacent cell bodies.) The mature striatum contains both striosome and matrix compartments (Graybiel and Moratalla, 1989; Gerfen, 1992). Figure 1 provides evidence that fetal DA fibers, on arrival in the mature striatum and having traveled a long distance, chose first to innervate in a patchy pattern. It is unclear whether the patchy innervation pattern formed by the transplant's DA fibers coincides with the host's compartmentations; however, it does suggest that there are preferred areas of dopaminergic innervation by the transplants.

\section{Reconstruction of degenerated connections}

There are two areas that need to be addressed regarding connectivity. First, the reinnervation of DA fibers may be attributable to a sprouting or regeneration of residual nigra DA neurons (Bohn et al., 1987; Kordower et al., 1991; Wang et al., 1991). Second, projections from the striatum to the transplanted mesencephalic graft may exist. Based on HRP tracing and immunocytochemical identification, the DA reinnervation is largely derived from the transplant, because many HRP-labeled TH-positive neurons were observed in the graft and not in the host's SN. The striatal neurons that did project to the $\mathrm{SNr}$ were readily seen invading the transplant in the nigral vicinity. Many HRP-antegradely filled fibers 
could be traced from the striatum into the internal capsule bundle and into the medial forebrain bundle, ending in the transplant in the SN. There were a great number of HRP-positive terminal punctates that were not seen in either the methyl greencounterstained neurons or in the TH-positive fibers, indicating that these punctates are not retrogradely transported HRP within the transplanted neurons but are the antegradely transported HRP striatal neuronal terminals.

Three other unique anatomical and neurochemical features were observed in the bridged transplant. First, a dual innervation of the striatonigral cell bodies and terminals was achieved for the first time with the current bridged transplantation. In the normal intact pathway, the nigral DA neurons innervate and regulate the striatonigral neurons in two places: the cell bodies at the striatum and the terminals at the SNr (for review, see Gerfen, 1992; Robertson, 1992). Sophisticated motor regulation may rely on this dual DA innervation to ensure the output of the striatal neurons, a key disinhibitor along the circuit for the excitatory thalamic action (Robertson, 1992). 6-OHDA lesioning in the SN disrupted both DA innervation of the striatal cell bodies and striatal terminals projecting to the SNr. Replenishment of DA innervation in the SN alone can reduce motor deficits (Nikkhak et al., 1994). The current method of bridged transplantation enables the graft to reach both the striatum (to regulate striatonigral cell bodies) and the $\mathrm{SNr}$ (to regulate striatonigral terminals). In turn, the grafted DA neurons have an opportunity to be regulated by afferents from the host's striatum (Fig. 3). Hence, regulation of DA release from the bridge-transplanted DA neurons may more closely mimic normal regulation of DA release. Second, a distinct difference was seen between this transplant method and the conventional ectopic target regional transplants in that DA released in the striatum from the bridged transplant was predominately from the terminals, because the somas and the dendrites were in the distant nigral regions. Third, the immunocytochemical staining of DA fibers in the dorsal and ventral striatum was well correlated with the neurochemical activity of DA. The distribution of TH-positive fiber density and DA release (uptake as well) showed a decreasing gradient from the bridged track to the distal regions (compare Figs. 1 and 4), indicating that release corresponds highly to the density of the TH-positive fibers around the fiber track. The corresponding gradients of DA release and clearance not only confirm the DA reinnervation but also indicate a restoration of neurochemical function and a regulated DA physiology. In addition to the above features, we also noticed that in the current KA-bridged transplant, DA release (at 6-8 weeks after transplantation) was achieved in a broader area in the striatum than observed previously in ectopic transplants of nigral tissue into the striatum after 3 months (Wang et al., 1994).

To generate a complete repair of a damaged circuit, the following criteria should be considered: (1) the reestablishment of both afferent and efferent anatomical connections, (2) the reformation of synaptic contacts, (3) the release of neurotransmitters and the clearance of extracellular transmitters, (4) the regeneration of electrophysiological activities, (5) the reversal of receptor supersensitivity (if it exists), and (6) the recuperation of behavioral deficit(s). The current study demonstrates that the bridged transplantation of mesencephalic tissue into the adult $\mathrm{SN}$ is capable of sending DA fibers through an artificial pathway to reinnervate the striatum in a manner recapitulating normal development. The DA fibers also extended on a small scale into the host's SNr. Thus, efferent connections to the host's striatonigral neuronal cell bodies in the striatum as well as to their axonal terminals in the $\mathrm{SNr}$ are achievable. The host's striatal neurons in turn innervated the graft, establishing a major afferent connection. Whereas synaptic contacts require an ultrastructural examination, neurotransmitter release at or near the synaptic sites has been demonstrated (Fig. 3). We and others have demonstrated that 6-OHDA lesions reduced or abolished $\mathrm{KCl}$-evoked DA release and DA clearance in the striatum (Castaneda et al., 1990; van Horne et al., 1992). We found that after bridged transplantation into the $\mathrm{SN}$, the reinnervating DA fibers can restore DA release on $\mathrm{K}^{+}$stimulation and decrease DA concentration after exogenous application of DA in the striatum. Such reestablished transmission is functionally effective as indicated by the rotational behavior data. This artificial pathway fulfills some basic requirements of a normal neuronal pathway.

\section{Behavior}

With the transplant in the SN and the bridged fibers innervating the striatum, the degree of body distortions and the number of turning behaviors were reduced in response to amphetamine challenge. The contralateral turnings seem to be a transient phenomenon in the early stage of the transplant. They could be a result of the combined function of the amount of DA release and the degree of DA receptor supersensitivity and also might reflect the balance between the DA and cortical inputs to the striatum and D1/D2/NMDA receptors/c-fos expression. Similar observations have also been described in other laboratories (Rioux et al., 1991; Cenci and Björklund, 1994). No rotational behavior improvement was observed in the control rats, which had few THpositive fibers reinnervating the striatum or SNr. This suggests that reinnervation to the striatum and/or nigra is essential for the current functional changes, and KA injection per se or misplaced transplants did not account for the functional amelioration. Furthermore, our unpublished data indicate that the limited KA damage of host neurons along the injection track did not seem to either improve or worsen the amphetamine-induced turning behavior or have any observable behavioral consequence. Detailed neurological-behavioral analysis will be done in the future.

The development and time course of the functional restoration are interesting phenomenon and should be closely screened in a long-term study. The rotational behavior test currently used is actually a crude functional test of locomotion and leaves many sophisticated and skillful movements to be examined in the future using the current model. However, the resumption of DA release and uptake as well as the reduction of ipsilateral and the appearance of contralateral turning behaviors together indicate that the current bridged transplantation rendered a DA innervation with functional consequences.

In conclusion, the current study demonstrates a breakthrough in generating a neurochemically and neuroanatomically efficient artificial pathway. This KA-bridged transplant opens up a feasible way to reconstruct a damaged neural pathway in the brain. Whether the artificial pathway can mimic the natural pathway requires further and extensive examination.

\section{REFERENCES}

Azmitia EC, Björklund A (1987) Cell and tissue transplantation into the adult brain. Ann NY Acad Sci 495.

Ballarin M, Ernfors P, Lindefors N, Persson H (1991) Hippocampal damage and kainic acid injection induce a rapid increase in mRNA for BDNF and NGF in the rat brain. Exp Neurobiol 114:35-43.

Björklund A, Stenevi U (1985) Neural grafting in the mammalian CNS. Amsterdam: Elsevier. 
Bohn MC, Cupti L, Marciano F, Gash DM (1987) Adrenal medulla grafts enhance recovery of striatal dopaminergic fibers. Science 237:913-916.

Castaneda E, Whishaw IQ, Robinson TE (1990) Changes in striatal dopamine neurotransmission assessed with microdialysis following recovery from a bilateral 6-OHDA lesion: variation as a function of lesion size. J Neurosci 10:1847-1854.

Cenci MA, Björklund A (1994) Transection of corticostriatal afferents abolishes the hyperexpression of Fos and counteracts the development of rotational overcompensation induced by intrastriatal dopamine-rich grafts when challenged with amphetamine. Brain Res 665:167-174.

Choi DW (1994) Glutamate receptors and the induction of excitotoxic neuronal death. Prog Brain Res 100:47-51.

Dunnett SB, Richards SJ (1990) Prog Brain Res 82. In: Neural transplantation: from molecular basis to clinical applications. New York: Elsevier.

Freed CR, Breeze RE, Rosenberg NL, Schneck SA, Kriek E, Qi JX, Lone T, Zhang YB, Snyder JA, Wells TH (1992) Survival of implanted fetal dopamine cells and neurologic improvement 12 to 46 months after transplantation for Parkinson's disease. N Engl J Med 327:1589-1592.

Freed WJ (1993) Neural transplantation: a special issue. Exp Neurol 122:1-4.

Gash DM, Sladek JR (1988) Prog Brain Res. 78. In: Transplantation into the mammalian CNS. Amsterdam: Elsevier.

Gerfen CR (1992) The neostriatal mosaic: multiple levels of compartmental organization. Trends Neurosci 15:133-139.

Gerhardt GA, Palmer MR (1987) Characterization of the techniques of pressure ejection and microiontophoresis using in vivo electrochemistry. J Neurosci Methods 22:147-159.

Gerhardt GA, Oke AF, Nagy G, Moghaddam B, Adams RN (1984) Nafion-coated electrodes with high selectivity for CNS electrochemistry. Brain Res 290:390-395.

Graybiel AM, Moratalla R (1989) Dopamine uptake sites in the striatum are distributed differentially in striosome and matrix compartments. Proc Natl Acad Sci USA 86:9020-9024.

Hudson JL, Levin DR, Hoffer BJ (1993) A 16-channel automated rotometer system for reliable measurement of turning behavior in 6-hydroxydopamine lesioned and transplanted rats. Cell Transplant 2:507-514.

Humpel C, Lindqvist E, Soderstrom S, Kylberg A, Ebendal T, Olson L (1995) Monitoring release of neurotrophic activity in the brains of awake rats. Science 269:552-554.

Kordower JH, Sanberg PR (1995) Neural transplantation into the CNS in cell transplantation, Vol 4. Amsterdam: Elsevier.

Kordower JH, Cochran E, Penn RD, Goetz C (1991) Putative chromaffin cell survival and enhanced host-derived TH-fiber innervation following a functional adrenal medulla autograft for Parkinson's disease. Ann Neurol 29:405-412.

Kordower JH, Freeman TB, Snow BJ, Vingerhoets FJ, Mufson EJ, Sanberg PR, Hauser R, Smith DA, Nauert GM, Perl DP (1995) Neuropathological evidence of graft survival and striatal reinnervation after the transplantation of fetal mesencephalic tissue in a patient with Parkinson's disease. N Engl J Med 27:1118-1124.

Lindvall O, Odin P (1994) Clinical application of cell transplantation and neurotrophic factors in CNS disorders. Curr Opin Neurobiol 4:752-757.

Lindvall O, Brundin P, Widner H, Rehncrona S, Gustaviii B, Frackowiak R, Leenders KL, Sawle g Roghwell JC, Marsden CD (1990) Grafts of fetal dopamine neurons survive and improve motor function in Parkinson's disease. Science 247:574-577.

Madrazo I, Franco-Bourland R, Aguilear M, Ostrosky-Solis F, Madrazo M, Cuevas C, Catrejon H, Guizar-Zahagun G, Magallon E (1991)
Autologous adrenal medullary, fetal mesencephalic, and fetal adrenal brain transplantation in Parkinson's disease: a long-term postoperative follow-up. J Neural Transplant Plast 2:157-164.

Mesulam M-M (1978) Tetramethyl benzidine for horseradish peroxidase neurohistochemistry: a non-carcinogenic blue reaction product with superior sensitivity for visualizing neural afferents and efferents. J Histochem Cytochem 26:106-117.

Nikkhak G, Cunningham MG, Jödicke A, Knappe U, Björklund A (1994) Improved graft survival and striatal reinnervation by microtransplantation of fetal nigral cell suspensions in the rat Parkinson model. Brain Res 633:133-143.

Pritzel M, Isacson O, Brundin P, Wiklund L, Björklund A (1986) Afferent and efferent connections of striatal grafts implanted into the ibotenic acid lesioned neostriatum in adult rats. Exp Brain Res 65:112-126.

Redmond Jr DE, Roth RH, Spencer DD, Naftolin F, Leranth C, Robbins RJ, Marek KL, Elsworth JD, Taylor JR, Sas KJ, et al (1993) Neural transplantation for neurodegenerative diseases: past, present, and future. Ann NY Acad Sci 695:258-266.

Rioux L, Gaudin DP, Bui LK, Grégoir L, Dipaolo T, Bédard PJ (1991) Correlation of functional recovery after a 6-hydroxydopamine lesion with survival of grafted fetal neurons and release of dopamine in the striatum of the rat. Neuroscience 40:123-131.

Robertson HA (1992) Dopamine receptor interactions: some implications for the treatment of Parkinson's disease. Trends Neurosci 15:201-206.

Rosenstein JM (1993) New horizons in neurotransplantation. Cell Transpl 2:183-266.

Takashima H, Walker BR, Cannon-spoor HE, Freed WJ (1993) Kainic acid lesions increase reafferentation of the striatum by substantia nigra grafts. Brain Res 621:71-78.

van Horne C, Hoffer BJ, Stromberg I, Gerhardt GA (1992) Clearance and diffusion of locally applied dopamine in normal and 6-hydroxydopamine-lesioned rat striatum. J Pharmacol Exp Ther 263:1285-1292.

Wang J, Bankiewicz KS, Plunkett RJ, Sheng J, Jacobwit DM (1991) Intracerebral transplantation in movement disorders. Amsterdam: Elsevier.

Wang Y, Wang SD, Lin SZ, Lui JC (1994) Restoration of dopamine overflow and clearance from the 6-hydroxydopamine lesioned rat striatum reinnervated by fetal mesencephalic grafts. J Pharmacol Exp Ther 270:814-821.

Zafra F, Hengerer B, Leibrock J, Thoenen H, Lindholm D (1990) Activity dependent regulation of BDNF and NGF mRNAs in the rat hippocampus is mediated by non-glutamate receptors. EMBO J 9:3545-3550.

Zhou FC, Buchwald N (1989) Connectivities of the striatal grafts in adult rat brain: a rich afference and scant striatonigral efference. Brain Res 504:15-30.

Zhou FC, Chiang YH (1995) Excitochemical-induced trophic bridging directs axonal growth of transplanted neurons to distal target. Cell Transplant 4:103-112.

Zhou FC, Bledsoe S, Murphy JM (1991) Serotonergic sprouting is induced by dopamine-lesion in substantia nigra of adult rat brain. Brain Res 556:108-116.

Zhou FC, Zhang JK, Lumeng L, Li TK (1994) Mesolimbic dopamine system in alcohol-preferring rats. Alcohol 12:403-412.

Zhou FC, Azmitia EC, Bledsoe S (1995) Rapid serotonergic fiber sprouting in response to ibotenic acid lesion in the striatum and hippocampus. Dev Brain Res 84:89-98. 